\title{
Abnormal functional connectivity of the posterior cingulate cortex is associated with depressive symptoms in patients with Alzheimer's disease
}

This article was published in the following Dove Press journal:

Neuropsychiatric Disease and Treatment

II October 2017

Number of times this article has been viewed

\author{
Jiangtao Zhang ${ }^{1,2}$ \\ Zhongwei Guo ${ }^{2}$ \\ Xiaozheng $\mathrm{Liu}^{3}$ \\ Xize Jia ${ }^{4}$ \\ Jiapeng $\mathrm{Li}^{2}$ \\ Yaoyao $\mathrm{Li}^{1,5}$ \\ Danmei Lv'1,5 \\ Wei Chen ${ }^{1,5}$
}

'Department of Psychiatry, Sir Run Run Shaw Hospital, Zhejiang University School of Medicine and the Collaborative Innovation Center for Brain Science, Hangzhou, Zhejiang, China; ${ }^{2}$ Tongde Hospital of Zhejiang

Province, Hangzhou, Zhejiang,

China; ${ }^{3}$ China-USA Neuroimaging

Research Institute \& Department

of Radiology, the Second Affiliated

Hospital, Wenzhou Medical University,

Wenzhou, China; ${ }^{4}$ Center for

Cognitive Brain Disorders \& Zhejiang

Key Laboratory for Research in

Assessment of Cognitive Impairments,

Hangzhou Normal University,

Hangzhou, China; ${ }^{5}$ Key Laboratory

of Medical Neurobiology of Chinese

Ministry of Health, Hangzhou,

Zhejiang, China
Correspondence: Wei Chen

Department of Psychiatry, Sir Run Run

Shaw Hospital, Zhejiang University

School of Medicine, and the Collaborative

Innovation Center for Brain Science,

3 East Qingchun Road, Hangzhou,

Zhejiang 310016 , China

Tel +8657I 86006375

Fax +86 57। 8788 780 I

Email srrcw@zju.edu.cn
Background: Depressive symptoms are significant and very common psychiatric complications in patients with Alzheimer's disease (AD), which can aggravate the decline in social function. However, changes in the functional connectivity (FC) of the brain in AD patients with depressive symptoms (D-AD) remain unclear.

Objective: To investigate whether any differences exist in the $\mathrm{FC}$ of the posterior cingulate cortex (PCC) between D-AD patients and non-depressed AD patients (nD-AD).

Materials and methods: We recruited $15 \mathrm{D}-\mathrm{AD}$ patients and 17 age-, sex-, educational level-, and Mini-Mental State Examination (MMSE)-matched nD-AD patients to undergo tests using the Neuropsychiatric Inventory, Hamilton Depression Rating Scale, and 3.0T resting-state functional magnetic resonance imaging. Bilateral PCC were selected as the regions of interest and between-group differences in the PCC FC network were assessed using Student's $t$-test.

Results: Compared with the nD-AD group, D-AD patients showed increased PCC FC in the right amygdala, right parahippocampus, right superior temporal pole, right middle temporal lobe, right middle temporal pole, and right hippocampus (AlphaSim correction; $P<0.05$ ). In the $\mathrm{nD}-\mathrm{AD}$ group, MMSE scores were positively correlated with PCC FC in the right superior temporal pole and right hippocampus (false discovery rate corrected; $P<0.05$ ).

Conclusion: Differences were detected in PCC FC between $\mathrm{nD}-\mathrm{AD}$ and D-AD patients, which may be related to depressive symptoms. Our study provides a significant enhancement to our understanding of the functional mechanisms underlying D-AD.

Keywords: Alzheimer's disease, depressive symptoms, resting-state functional MRI, PCC

\section{Introduction}

Alzheimer's disease (AD) is a progressive neurodegenerative disorder characterized by a decline in cognitive function, including the progressive loss of memory, reasoning, and language. Furthermore, almost all AD patients are affected by neuropsychiatric symptoms over the course of their illness. ${ }^{1,2}$ Depressive symptoms are very common and significant psychiatric complications are known to affect $30 \%-50 \%$ of AD patients. ${ }^{3}$ These complications can increase the burden upon AD patients and their caregivers, become a trigger for further disability, and shorten lifespan. ${ }^{3,4}$ However, little is known about the underlying mechanisms of depressive symptoms in AD patients.

Morphological studies of the brain, using magnetic resonance imaging (MRI), have revealed cortical thinning in the temporal, parietal, ${ }^{5}$ and frontal regions ${ }^{6,7}$ of AD patients with depressive symptoms (D-AD). Single photon emission computed tomography studies have also shown that the brains of people with D-AD exhibit hypoperfusion in the bilateral cingulate gyri and precuneus, ${ }^{8}$ left inferior frontal region, ${ }^{9}$ 
right orbitofrontal cortex, inferior frontal gyri, ${ }^{10}$ and the left frontal cortex. ${ }^{11}$ Some positron emission tomography (PET) studies have also revealed hypo-metabolism in the dorsolateral prefrontal regions ${ }^{12,13}$ and in the left anterior cingulate cortices in D-AD patients. ${ }^{14}$ Furthermore, a PETMRI study showed that, compared with non-depressed participants, $\beta$-amyloid-positive depressed mild cognitive impairment (MCI) patients had higher amyloid loading in the fronto-temporal and insular cortices, with coincident hyper-metabolism in the frontal cortices. ${ }^{15}$

Collectively, these studies indicate that the structure and function of the brain in D-AD patients is abnormal; however, these studies were limited to only a few specific brain regions. Recent network studies have demonstrated that normal global functional organization is disrupted in the AD brain, affecting both local connectivity and critical long-distance connections and specialized hub nodes. ${ }^{16-18}$ Resting-state functional MRI (fMRI) is a useful tool for studying intrinsic functional connectivity (FC). Using this approach, Guo et al observed reduced regional coherence in the right precentral gyri, right superior frontal gyri, right middle frontal gyri, and the right inferior frontal gyri in D-AD patients compared to non-depressed AD (nD-AD) patients. ${ }^{19}$ To investigate the intrinsic pattern of poor connectivity in whole-brain functional networks of D-AD patients, Guo et al used degree centrality at the voxel level. Their experiments found that D-AD patients possessed lower values in the right middle frontal, precentral, and postcentral gyrus, compared with $\mathrm{nD}$-AD patients. Seed-based analysis, which is also based on abnormal degree centrality, further suggested that the connectivity between the precentral, postcentral, supplementary motor area, and the middle cingulum was reduced. ${ }^{20}$

However, to date, there have been no studies of the default mode network (DMN) characteristics of D-AD patients. The DMN includes the medial prefrontal cortex, medial temporal lobes, dorsolateral prefrontal cortex, posterior cingulate cortex (PCC), and inferior parietal lobes. ${ }^{21,22}$ The DMN is considered to be involved in the process of self-referential processing, affective cognition, and emotion regulation. Poor functional connectivity in the DMN has been widely reported in a variety of mental disorders, including major depressive disorder (MDD). ${ }^{23}$ In MDD patients, it has also been reported that during emotional processing, several sub-regions of the DMN show significant reductions in negative blood oxygenation level-dependent responses, which correlate with the severity of depression and feelings of hopelessness. ${ }^{24}$ A resting-state FC study demonstrated that the FC between the PCC and the right medial temporal lobe were significantly stronger during rest, but significantly weaker during task performance, in remitted late-onset depression (rLOD) patients. ${ }^{25}$ Accordingly, it is possible that the DMN may be the primary neural network underlying the complex relationships between depressive symptoms.

We hypothesized that depressive symptoms can affect FC of the DMN in AD patients. Zhong et al reported that the PCC is a convergence center and may represent the only convergence region receiving interactions from other regions in the DMN. ${ }^{26}$ Therefore, we employed resting-state fMRI to explore differences in $\mathrm{FC}$ between $\mathrm{D}-\mathrm{AD}$ and $\mathrm{nD}-\mathrm{AD}$ patients, with PCC as the specific region of interest (ROI). This study will help to contribute to our wider understanding of the mechanisms underlying D-AD.

\section{Materials and methods Participants}

In total, 32 patients, aged between 65 and 80 years, were recruited between February 2013 and December 2014 from Tongde Hospital in Zhejiang Province, China. All participants were right-handed and had received more than 6 years of schooling. All participants met criteria for the diagnosis of probable $\mathrm{AD}$ according to the recommendations of the National Institute on Aging-Alzheimer's Association workgroup's diagnostic guidelines for Alzheimer's disease: ${ }^{27}$ a score of 20-24 on the Mini-Mental State Examination (MMSE), and a score of 1 on the Clinical Dementia Rating scale. Depressive symptoms met the Diagnostic and Statistical Manual of Mental Disorders (4th Edition), ${ }^{28}$ which meant that all D-AD patients had one or more of the two core criteria (depressed mood and loss of interest or pleasure), for a duration of at least 2 weeks. The severity of depressive symptoms was assessed by the 17-item Hamilton Depression Rating Scale (HAMD-17). ${ }^{29}$ The Neuropsychiatric Inventory (NPI) is a valid and reliable instrument for measuring the noncognitive symptoms of dementia ${ }^{30,31}$ and was used to examine other neuropsychiatric conditions aside from depression: ${ }^{30}$ D-AD scored 1 on the depression domain, and scored 0 on each of the other eleven NPI domains. The total score on the depression domain of the NPI (D-NPI) represents the product of the frequency (scores 1-4) and severity (scores 1-3). Based on recommendations for clinical trial entry criteria, D-NPI scores of $\geq 4$ indicate clinical significance. ${ }^{32}$ All of these scales were assigned by trained neuropsychologists.

Patients with a history of significant visual or hearing impairments were excluded, since this rendered interview participation difficult. Participants with a history of other neurological disorders, mental illnesses, or significant alcohol, 
psychotropic medications, and/or other substance abuse, were also excluded. We also excluded subjects whose dual-echo MR images showed two or more hyper-intense lesions with diameters $\geq 5 \mathrm{~mm}$, or more than four hyper-intense lesions with diameters between 0 and $5 \mathrm{~mm}$.

\section{MRI data acquisition}

All participants were scanned using a $3 \mathrm{~T}$ Siemens scanner (Magnetom Verio; Siemens, Erlangen, Germany) with a 12-channel head coil. Resting-state functional images were acquired with a gradient-echo echo-planar imaging (EPI) sequence: repetition time (TR)/echo time $(\mathrm{TE})=2,000 / 30 \mathrm{~ms}$, flip angle $=90^{\circ}, 33$ axial slices, thickness $/$ gap $=4.8 / 0 \mathrm{~mm}$, matrix $=64 \times 64$, and field of view $($ FOV $)=200 \times 200 \mathrm{~mm}^{2}$. Each condition consisted of 200 functional volumes. Subsequently, high-resolution structural images were obtained using a magnetization-prepared rapid gradient-echo sequence: TR/ TE/inversion time $(\mathrm{TI})=1,900 / 2.48 / 900 \mathrm{~ms}$, flip angle $=9^{\circ}, 128$ sagittal slices, slice thickness $/$ gap $=1 / 0 \mathrm{~mm}$, matrix $=512 \times 512$, and $\mathrm{FOV}=256 \times 256 \mathrm{~mm}^{2}$. The participants were informed to lie quietly, keep their eyes closed, and not to fall asleep. Scanner noise was attenuated with earplugs and head motion was restricted with foam padding around the head.

\section{Image analysis}

\section{Image pre-processing}

Resting-state fMRI data were processed using Statistical Parametric Mapping 8 (SPM, http://www.fil.ion.ucl.ac.uk/ spm) and Data Processing Assistant for Resting-State fMRI (http://www.restfmri.net). ${ }^{33}$ The procedure included the following pre-processing steps: 1) removal of the first ten volumes of functional images because of the instability of the initial signal and to allow participants to adapt to the scanner environment; 2) slice timing for acquisition delay between slices; 3 ) head motion correction, with all participants having $<1.5 \mathrm{~mm}$ maximum displacement in $\mathrm{x}, \mathrm{y}$, or $\mathrm{z}$ planes and $1.5^{\circ}$ of angular motion during the entire fMRI scan; 4) spatial normalization (realigned functional data were normalized to the standard EPI template in the Montreal Neurological Institute space by using 12 parameter affine transformation and non-linear deformation) and resampling of functional images to $3 \mathrm{~mm}$ isotropic voxels; 5) removal of linear trends within the time series; and 6) band-pass filtering $(0.01-0.08 \mathrm{~Hz})$ to reduce low-frequency drift and physiological high-frequency respiratory and cardiac noise. Finally, several sources of spurious variances were removed by linear regression, including six head motion parameters, along with average signals from cerebrospinal fluid and white matter. ${ }^{34}$

\section{Analysis of functional connectivity}

We selected the bilateral PCC, consisting of Brodmann areas 29, 30, 23, and 31, as the template ROI using Wake Forest University Pick Atlas software. ${ }^{35,36}$ In order to perform ROIbased correlation analysis, the mean PCC signal intensity was calculated by averaging the time series of all voxels within a selected ROI. The resulting time course was then used to perform Pearson linear correlation analysis with all voxels of the brain data. ${ }^{37,38}$ A Fisher z-transformation was applied to improve normality of the correlation coefficients. ${ }^{34,37}$

\section{Statistical analysis}

Differences in demographic and clinical data between the $\mathrm{D}-\mathrm{AD}$ and $\mathrm{nD}-\mathrm{AD}$ groups of patients were analyzed using two-sample Student's $t$-tests and $\chi^{2}$-tests.

To investigate differences in PCC connectivity between the two groups of patients, two-sample $t$-tests were performed using REST (http://www.restfmri.net). AlphaSim, a program based on Monte Carlo simulation and implemented in Analysis of Functional Neuro Images (http://afni.nimh.nih.gov), was used for multiple comparison correction. Monte Carlo simulations determine the random cluster size distribution for a given per voxel threshold. ${ }^{39}$ According to this distribution, a corrected $P$-value less than 0.05 had obtained a combination criterion of voxels with a $P$-value $\leq 0.05$ and a cluster size $\geq 198$ voxels within the intersection mask.

We then investigated correlations between PCC FC and clinical symptoms. Correlation analyses were then performed between the two groups of patients in terms of PCC FC and MMSE, NPI, and HAMD scores. The threshold for statistical significance was set at false discovery rate corrected $P<0.05$ in SPM8.

\section{Results \\ Demographics and neuropsychological tests}

There was no significant difference between the D-AD and $\mathrm{nD}$-AD groups in terms of age, sex distribution, and levels of education. The MMSE was not statistically different between the groups and no subjects were excluded by our exclusion criteria. As expected, there was a significant difference in HAMD and D-NPI scores when compared between the two groups. Further details are presented in Table 1.

\section{Between-group analyses of PCC connectivity}

Between-group analyses were carried out using REST two-sample $t$-tests $(P<0.05$, AlphaSim corrected; Table 2). 
Table I Demographic and clinical characteristics of the study participants

\begin{tabular}{lllll}
\hline & $\begin{array}{l}\text { D-AD } \\
(\text { mean } \pm \text { SD) }\end{array}$ & $\begin{array}{l}\text { nD-AD } \\
(\text { mean } \pm \text { SD) }\end{array}$ & t $\chi^{2}$ & P-value \\
\hline Age (years) & $73.5 \pm 4.73$ & $75.8 \pm 3.75$ & -1.564 & 0.128 \\
Sex, N (M:F) & $15(6: 9)$ & $17(8: 9)$ & 0.161 & 0.735 \\
Educational & $8.6 \pm 1.83$ & $8.5 \pm 1.84$ & 0.211 & 0.835 \\
level (years) & & & & \\
HAMD & $13.9 \pm 2.31$ & $2.82 \pm 1.91$ & 14.872 & 0.000 \\
D-NPI & $6.6 \pm 1.92$ & 0 & 14.206 & 0.000 \\
MMSE & $19.6 \pm 2.20$ & $21.0 \pm 2.09$ & -1.845 & 0.075 \\
\hline
\end{tabular}

Abbreviations: D-AD, Alzheimer's disease patients with depressive symptoms; $n D-A D$, non-depressed Alzheimer's disease patients; $M$, male; $F$, female; HAMD, Hamilton Depression Rating Scale; D-NPI, depression domain of the Neuropsychiatric Inventory; MMSE, Mini-Mental State Examination.

This allowed us to determine the between-group difference in PCC connectivity. We then compared the D-AD group with the $\mathrm{nD}-\mathrm{AD}$ group and showed that connectivity between the PCC and the right amygdala, right parahippocampal gyri, right superior temporal pole, right middle temporal lobe, right middle temporal pole, and right hippocampus was increased in the D-AD group (Table 2, Figure 1).

\section{Correlations between PCC FC and clinical symptoms}

Regarding the $\mathrm{D}-\mathrm{AD}$ patients, a correlation analysis between the PCC FC of the right amygdala, right parahippocampal gyri, right superior temporal pole, right middle temporal lobe, right middle temporal pole, right hippocampus, and the MMSE, NPI, and HAMD scores suggested that the MMSE scores of the $\mathrm{nD}$-AD group were positively correlated with PCC FC in the right superior temporal pole and right hippocampus (Table 3). There were no significant correlations between the PCC FC of the right amygdala, right

Table 2 Brain regions with significantly increased functional connectivity in the D-AD group compared with the $\mathrm{nD}-\mathrm{AD}$ group (AlphaSim correction, $P<0.05$ )

\begin{tabular}{lllllll}
\hline Brain regions & BA & Vols & $\begin{array}{l}\text { Stereotaxic } \\
\text { Coordinates, } \\
\end{array}$ & & \multicolumn{5}{c}{$\begin{array}{l}\text { Peak } \\
\text { intensity }\end{array}$} \\
\cline { 3 - 6 } & & & $\mathbf{x}$ & $\mathbf{y}$ & $\mathbf{z}$ & \\
\hline R Amygdala & $34 / 36$ & 18 & 27 & 3 & -27 & $4.777 \mid$ \\
R Parahippocampal & $28 / 36$ & 34 & 28 & 3 & -29 & 4.22891 \\
R Temporal_Pole_Sup & $21 / 38 / 48$ & 46 & 47 & 7 & -14 & 2.82391 \\
R Temporal_Mid & 21 & 31 & 50 & -1 & -14 & 2.37799 \\
R Temporal_Pole_Mid & 21 & 31 & 47 & 7 & -22 & 2.36318 \\
R Hippocampus & 20 & 35 & 33 & -23 & -8 & 2.31557 \\
\hline
\end{tabular}

Abbreviations: D-AD, Alzheimer's disease patients with depressive symptoms; nD-AD, non-depressed Alzheimer's disease patients; BA, Brodmann area; Vols, number of voxels; $R$, right. parahippocampal gyri, right superior temporal pole, right middle temporal lobe, right middle temporal pole, or right hippocampus and NPI and HAMD scores in the $\mathrm{nD}-\mathrm{AD}$ group (Table 3). Furthermore, there were no significant correlations between the PCC FC of the right amygdala, right parahippocampal gyri, right superior temporal pole, right middle temporal lobe, right middle temporal pole, right hippocampus and MMSE, NPI, and HAMD scores in the D-AD group (Table 4).

\section{Discussion}

In this exploratory study, we employed resting-state fMRI to investigate different levels of FC in $\mathrm{D}-\mathrm{AD}$ patients compared with $\mathrm{nD}-\mathrm{AD}$ patients using the bilateral $\mathrm{PCC}$ as an ROI. Our results showed that in $\mathrm{D}-\mathrm{AD}$ patients, compared with $\mathrm{nD}-\mathrm{AD}$ patients, there was an increase in resting-state FC between the PCC and the right hippocampus, right parahippocampal gyri, right amygdala, and right temporal pole.

The hippocampus is the main structural substrate for episodic memory deficits ${ }^{40,41}$ and is among the first regions targeted by AD pathology. ${ }^{42,43}$ The hippocampus demonstrates predominantly diminished $\mathrm{FC}$ with other regions as $\mathrm{AD}$ progresses. ${ }^{44}$ As a core region in the limbic system, the hippocampus is involved in not only cognitive functions such as learning and memory, ${ }^{45}$ but also the regulation of motivation and emotion. ${ }^{46,47}$ Specifically, the hippocampus may potentially mediate the relationship between helplessness and depression by regulating gene expression. ${ }^{48,49}$ Altered FC in the hippocampus may be related to the deficits in emotionmediated memory formation observed in depression..$^{50,51}$ In this study, compared with nD-AD patients, the FC of the PCC and the hippocampus in D-AD patients was increased, which may reflect how a reduced ability to form memories arises from the effects of depressive symptoms. A previous neuropathological study also showed that D-AD leads to increased hippocampal plaques and tangles, and a more rapid cognitive decline, compared to $\mathrm{nD}-\mathrm{AD}$ patients. ${ }^{52}$ This further suggests that the reduced ability to form memories arises from the effects of depressive symptoms in D-AD patients.

In our study, we found increased FC between the PCC and the right parahippocampal gyrus in $\mathrm{D}-\mathrm{AD}$ patients. The parahippocampal gyrus is an important region of the limbic system and a key region in the episodic memory network. ${ }^{53,54}$ Imaging findings have consistently identified abnormal changes in the right parahippocampus, such as gray matter atrophy, ${ }^{55}$ reduced cortical thickness, ${ }^{56}$ and hypoperfusion, ${ }^{57}$ which are all related to cognitive degradation in $\mathrm{AD}$ patients. Chen et al also found that the right parahippocampal gyrus 


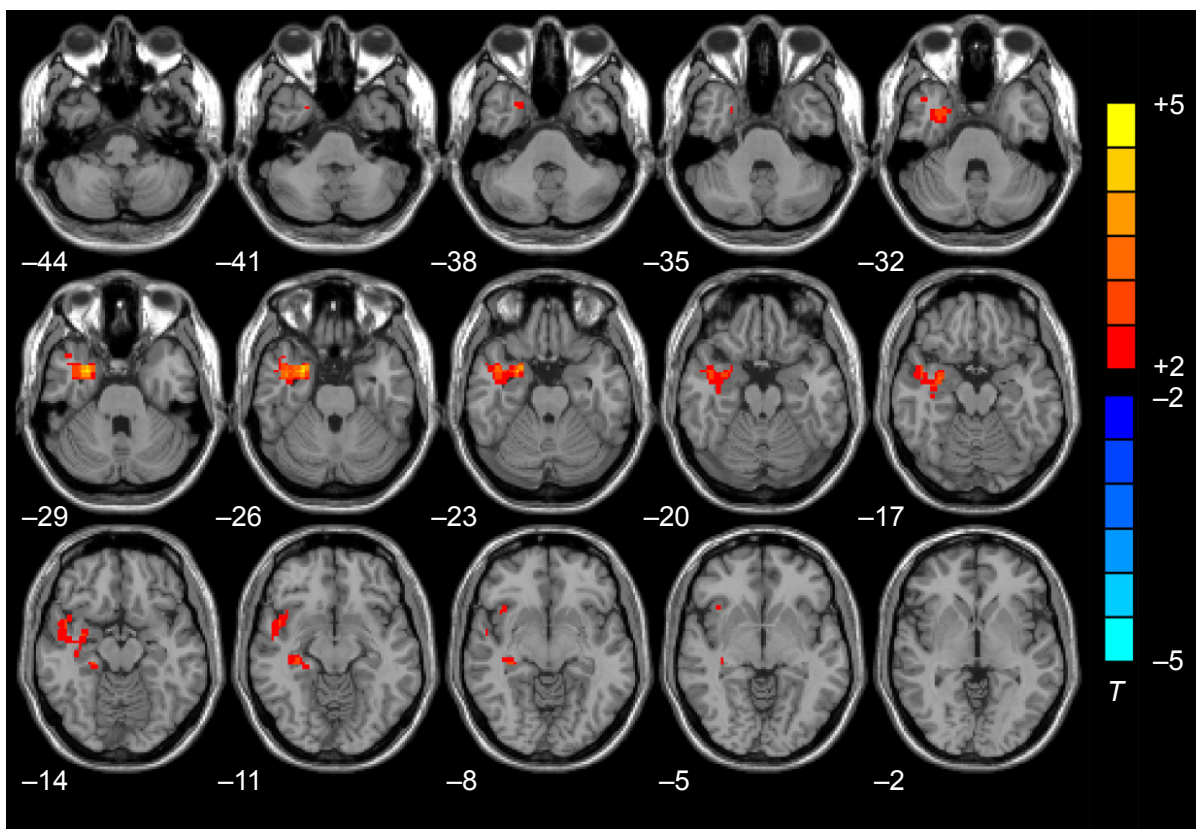

Figure I Brain regions showing increased PCC FC in the $\mathrm{nD}-\mathrm{AD}$ group compared with the D-AD group on an axial map ( $P<0.05$, AlphaSim corrected). Note: $T$-value scale is to the right of the image.

Abbreviations: PCC, posterior cingulate cortex; FC, functional connectivity; nD-AD, non-depressed Alzheimer's disease patients; D-AD, Alzheimer's disease patients with depressive symptoms.

showed imbalanced bidirectional effective connections in $\mathrm{AD}$ patients, which received weaker input connections from the neocortices, although output connections were significantly increased. ${ }^{58}$ The FC between the parahippocampal gyrus and the PCC is known to be reduced in AD patients and becomes more severely damaged as the disease progresses. ${ }^{59}$ Conversely, previous studies have detected gray matter reduction and diffusion tensor MRI abnormalities in the right parahippocampal gyrus in MDD. ${ }^{60,61}$ Remitted depressed participants are also known to show increased connectivity between the PCC and the parahippocampal gyri, compared with healthy controls. ${ }^{62}$ These studies indicate that the

Table 3 Correlation between FC and MMSE, NPI, and HAMD in the $\mathrm{nD}-\mathrm{AD}$ group (FDR corrected $P<0.05$ )

\begin{tabular}{|c|c|c|c|c|c|c|}
\hline \multirow[t]{2}{*}{ Brain regions } & \multicolumn{2}{|l|}{ MMSE } & \multicolumn{2}{|c|}{ D-NPI ${ }^{a}$} & \multicolumn{2}{|c|}{ HAMD } \\
\hline & $r$ & $P$-value & $r$ & $P$-value & $r$ & $P$-value \\
\hline Amygdala & 0.1400 & 0.5920 & - & - & 0.3789 & 0.1337 \\
\hline R Parahippocampal & 0.1187 & 0.6501 & - & - & 0.4055 & 0.1064 \\
\hline R Temporal_Pole_Sup & 0.4980 & 0.0419 & - & - & 0.2322 & 0.3699 \\
\hline R Temporal_Mid & 0.4165 & 0.0963 & - & - & 0.0638 & 0.8079 \\
\hline R Temporal_Pole_Mid & 0.4252 & 0.0899 & - & - & 0.2520 & 0.8079 \\
\hline R Hippocampus & 0.5334 & 0.0275 & - & - & 0.4574 & 0.0649 \\
\hline
\end{tabular}

Note: aBecause all the NPI scores were 0 in $\mathrm{nD}-\mathrm{AD}$ group, the correlation analysis was unable to be performed.

Abbreviations: FC, functional connectivity; MMSE, Mini-Mental State Examination D-NPI, depression domain of the Neuropsychiatric Inventory; HAMD, Hamilton Depression Rating Scale; nD-AD, non-depressed Alzheimer's disease patients; D-AD, Alzheimer's disease patients with depressive symptoms; R, right. structure and function of the parahippocampus are abnormal in both MDD and AD patients. Our present study showed increased FC between the PCC and the right parahippocampal gyrus in $\mathrm{D}-\mathrm{AD}$ patients compared with $\mathrm{nD}-\mathrm{AD}$ patients. In combination, these studies reflect the difference in changes of brain function between $\mathrm{D}-\mathrm{AD}$ and $\mathrm{nD}-\mathrm{AD}$ patients. This may represent the underlying reason for the deterioration of episodic memory in D-AD patients, which may be further affected by depressive symptoms.

The amygdala and temporal poles are important parts of the affective network, which includes the amygdala, orbitofrontal cortex, temporal cortex, pallidum, and insular cortex. ${ }^{63}$ These regions are involved in mood regulation and affective processing. ${ }^{64,65}$ It is believed that depressive mood might be correlated with the affective network. ${ }^{66}$ Dolan et al demonstrated responses of the amygdala and temporal poles to the activation of an emotional memory retrieval task. ${ }^{67}$ The amygdala is the most important area of the human brain for forming emotional memories and modulating emotional response and is considered to be a key brain region in relation to stress reactivity and vulnerability for depressive disorder. ${ }^{68}$ Increased activity of the amygdala has been reported in response to negative emotional signals (eg, faces, pictures, and words) in MDD. ${ }^{69,70}$ Ramasubbu et al found that the intrinsic connectivity of the amygdala with the temporal poles was increased in adults with MDD and that increased FC was 
Table 4 Correlation between FC and MMSE, NPI, and HAMD in the D-AD group (FDR corrected $P<0.05$ )

\begin{tabular}{|c|c|c|c|c|c|c|}
\hline \multirow[t]{2}{*}{ Brain regions } & \multicolumn{2}{|l|}{ MMSE } & \multicolumn{2}{|l|}{ D-NPI } & \multicolumn{2}{|c|}{ HAMD } \\
\hline & $r$ & $P$-value & $r$ & $P$-value & $r$ & $P$-value \\
\hline R Amygdala & -0.3896 & 0.1512 & 0.3209 & 0.2435 & 0.3568 & 0.1917 \\
\hline R Parahippocampal & -0.3762 & 0.1670 & 0.3104 & 0.2602 & 0.3175 & 0.2489 \\
\hline R Temporal_Pole_Sup & -0.1437 & 0.6095 & 0.2111 & 0.4501 & 0.1007 & 0.7211 \\
\hline R Temporal_Mid & -0.1073 & 0.7035 & 0.3105 & 0.2599 & 0.2459 & 0.3769 \\
\hline R Temporal_Pole_Mid & -0.0659 & 0.8156 & 0.2317 & $0.406 I$ & 0.3002 & $0.277 \mid$ \\
\hline R Hippocampus & 0.1663 & 0.5536 & 0.1617 & 0.5647 & 0.1709 & 0.5426 \\
\hline
\end{tabular}

Abbreviations: FC, functional connectivity; MMSE, Mini-Mental State Examination; D-NPI, depression domain of the Neuropsychiatric Inventory; HAMD, Hamilton Depression Rating Scale; D-AD, Alzheimer's disease patients with depressive symptoms; FDR, false discovery rate; R, right.

inversely correlated with symptom severity. ${ }^{71}$ Another two studies also showed increased amygdala resting-state FC with the dorsal mid-insular cortex ${ }^{72}$ and the sub-genual anterior cingulate cortex in patients with depression. ${ }^{73}$ Longitudinal alteration of amygdala FC with regions of the DMN which are involved in MCI were reduced, which was significantly correlated with cognitive ability. ${ }^{74}$ In $\mathrm{AD}$, the strengthening effect of emotion on memory is impaired, and the level of damage is associated with the level of amygdala damage and emotional projections. ${ }^{75}$ Yao et al found that reduced FC in $\mathrm{AD}$ patients was mainly located between the amygdala and the regions that were included in the DMN. Importantly, the reduced level of FC between the amygdala and some of the identified regions positively correlated with MMSE scores, which indicated that the impairment of cognitive function was related to alterations in the FC of the amygdala. ${ }^{76}$ The fact that our current study identified increased resting-state FC between the PCC and the amygdala in D-AD patients may, in part, be the basis for the differential clinical scenarios between $\mathrm{D}-\mathrm{AD}$ and $\mathrm{nD}-\mathrm{AD}$ patients. The more severe clinical condition of D-AD may partly arise from depressive symptoms affecting the amygdala and consequential modulation of emotional responses.

The temporal pole is the anterior-most portion of the temporal lobes and is considered to be part of an extended limbic system due to its location lateral to the amygdala, posterior to the orbital frontal cortex, and heavily interconnected to the limbic and paralimbic regions. ${ }^{77}$ The temporal pole is involved in numerous aspects of emotional processing, including the subjective perception of affect, ${ }^{78,79}$ as well as anxiety and stress. ${ }^{80,81}$ The function of the temporal pole is abnormal in MDD patients. Activation of the right anterior temporal pole in depressed individuals is increased when they attempt to voluntarily downregulate sad feelings. ${ }^{82}$ Guo et al directly examined cerebellar-DMN connectivity in drugnaive MDD patients by using the cerebellum Crus I as an ROI, and observed significantly increased FCs between the right Crus I and the superior temporal pole. ${ }^{83}$ Liu et al also demonstrated that depressed patients showed significantly increased FC between the cerebellum and the temporal poles ${ }^{84}$ Meanwhile, significantly reduced intrinsic connectivity in the anterior temporal pole was found in amnestic MCI patients, ${ }^{85}$ which reflected the effect of the temporal pole in cognition. The anterior temporal pole has also been associated with emotional memory retrieval. ${ }^{67}$ Our present results show that the resting-state $\mathrm{FC}$ of the right temporal pole, and the PCC in D-AD patients, was increased compared with $\mathrm{nD}-\mathrm{AD}$ patients. Based on these findings, we may assume that dysfunction of the temporal pole leads to abnormal emotional processing, which may further lead to depression and worsened emotional memory retrieval in $\mathrm{D}-\mathrm{AD}$ patients. Our study therefore provides significant insight into the important effects of depressive symptoms on emotional processing in the right temporal pole in $\mathrm{AD}$.

Increased FC has been noted between the PCC and the right medial temporal lobe, which contains parts of the hippocampus, parahippocampal gyrus, amygdale, and uncus during resting state in rLOD patients, and became significantly weaker during task performance. ${ }^{25}$ Previous studies of $\mathrm{AD}$ patients showed a correlation between episodic memory decline and abnormal structure and function of the medial temporal lobe. ${ }^{86,87}$ Our current results indicated that the increased resting-state FC between the PCC and right hippocampus, right parahippocampal gyri, right amygdala, and right temporal pole, may underlie the defects in memory and cognitive function in $\mathrm{D}-\mathrm{AD}$ patients. This may result from the decline of information relating to memory, episodic memory, emotional response, and emotional processing, which may be associated with the depressive symptoms.

The MMSE scores of the $\mathrm{nD}-\mathrm{AD}$ group in our study were positively correlated with the PCC FC in the right superior temporal pole and the right hippocampus, which further indicates that the FCs of the PCC, the hippocampus, and the temporal lobe are associated with memory loss in $\mathrm{AD} .^{88}$ 
However, in the D-AD group, there were no significant correlations between the PCC FC of the right amygdala, right parahippocampus, right superior temporal pole, right middle temporal lobe, right middle temporal pole, and right hippocampus and the symptoms. This may be related to our small sample size, or it may reflect the differential pathogenic mechanisms of $\mathrm{D}-\mathrm{AD}$ and $\mathrm{nD}-\mathrm{AD}$, which require further research.

\section{Limitations and future directions}

Although the results of this study provide new knowledge, limitations remain regarding our small sample size. According to Eklund et al ${ }^{89}$ our primary $P$-value, which we set for between-group analysis, was too stringent. When we utilized a voxel $P$-value of $P<0.001$, there were no significant differences identified between groups. This may have been due to our relatively small sample size. Consequently, in future studies, it may be wiser to use a less stringent $P$-value. However, reducing the stringency of our primary threshold may lead to false positive findings. Consequently, further studies are now needed to examine the reproducibility of the current study.

It is also possible that scanner variability had an effect upon our data. Some types of physiological noise, such as cardiac and respiratory rates, can affect the low-frequency domain of fMRI signals. ${ }^{90,91}$ However, cardiac and respiratory rates were not recorded during the scans in this study. Additional neuroimaging evidence, such as structural abnormalities, are now needed as a synthesized biomarker to ensure a more reliable clinical diagnosis of this complex disorder.

Furthermore, it is important to note that although neuropsychiatric symptoms are not required for the diagnosis of $\mathrm{AD}$, they represent a major clinical feature and contribute to the distress of both patients and caregivers. Currently, there is no gold standard source of information for the assessment of these conditions. In the current study, we employed the informant-based NPI to measure neuropsychiatric symptoms because of its long-standing use in the dementia literature, ease of administration, and good validity and reliability. ${ }^{92}$ However, it has been shown that the educational level and the psychological state of AD patients, and the attitude of their caregivers toward the disease, may influence the results of this inventory. ${ }^{93}$ These factors may introduce some degree of bias into our assessment by the over-reporting or under-reporting of symptoms, or variations in perception of behavior. ${ }^{93,94}$ Finally, we did not acquire pathological confirmation of our clinical diagnosis of early AD, although our diagnosis was based on detailed clinical examination, extensive neuropsychological testing, and structural imaging, and has been shown to achieve an accuracy of $85 \%-95 \% .^{95}$

\section{Conclusion}

To our knowledge this is the first study to report differences in the resting-state $\mathrm{FC}$ of $\mathrm{D}-\mathrm{AD}$ patients compared with $\mathrm{nD}-\mathrm{AD}$ patients using the bilateral PCC as the ROI. We identified abnormal FC in both the limbic system and the affective network of D-AD patients, which may have arisen from depression. Our study provides a significant step forward in our understanding of the neural mechanisms of D-AD.

\section{Ethics approval and consent to participate}

All participants (or their legal representatives) provided informed written consent. The research protocol was approved by the Ethics Committee of Tongde Hospital in Zhejiang Province.

\section{Acknowledgment}

This research was supported by the General Project of the Department of Science and Technology of Zhejiang Province (2015KYB073), the Traditional Chinese Medicine Science and Technology Plan of Zhejiang Province (2015ZA018), the Key Project of the Department of Science and Technology of Zhejiang Province (2007C13053, 2013C03045-5), National Key Technology R\&D Program (2015BAI13B01), and Major Scientific and Technological Innovation Projects in Hangzhou City (20131813A09) to Wei Chen.

\section{Disclosure}

The authors report no conflicts of interests in this work.

\section{References}

1. Drevets WC, Rubin EH. Psychotic symptoms and the longitudinal course of senile dementia of the Alzheimer type. Biol Psychiatry. 1989; 25(1):39-48.

2. Lyketsos CG, Sheppard JM, Steinberg M, et al. Neuropsychiatric disturbance in Alzheimer's disease clusters into three groups: the Cache County study. Int J Geriatr Psychiatry. 2001;16(11): 1043-1053.

3. Olin JT, Schneider LS, Katz IR, et al. Provisional diagnostic criteria for depression of Alzheimer disease. Am J Geriatr Psychiatry. 2002; 10(2):125-128.

4. Lou Q, Liu S, Huo YR, Liu M, Liu S, Ji Y. Comprehensive analysis of patient and caregiver predictors for caregiver burden, anxiety and depression in Alzheimer's disease. J Clin Nurs. 2015;24(17-18):2668-2678.

5. Lebedeva A, Westman E, Lebedev AV, et al. Structural brain changes associated with depressive symptoms in the elderly with Alzheimer's disease. J Neurol Neurosurg Psychiatry. 2014;85(8):930-935.

6. Son JH, Han DH, Min KJ, Kee BS. Correlation between gray matter volume in the temporal lobe and depressive symptoms in patients with Alzheimer's disease. Neurosci Lett. 2013;548:15-20. 
7. Hayata TT, Bergo FP, Rezende TJ, et al. Cortical correlates of affective syndrome in dementia due to Alzheimer's disease. Arq Neuropsiquiatr. 2015;73(7):553-560.

8. Liao YC, Liu RS, Lee YC, et al. Selective hypoperfusion of anterior cingulate gyrus in depressed AD patients: a brain SPECT finding by statistical parametric mapping. Dement Geriatr Cogn Disord. 2003; 16(4):238-244.

9. Honda H, Terada S, Sato S, et al. Subjective depressive mood and regional cerebral blood flow in mild Alzheimer's disease. Int Psychogeriatr. 2014;26(5):817-823.

10. Kang JY, Lee JS, Kang H, et al. Regional cerebral blood flow abnormalities associated with apathy and depression in Alzheimer disease. Alzheimer Dis Assoc Disord. 2012;26(3):217-224.

11. Kataoka K, Hashimoto H, Kawabe J, et al. Frontal hypoperfusion in depressed patients with dementia of Alzheimer type demonstrated on 3DSRT. Psychiatry Clin Neurosci. 2010;64(3):293-298.

12. Holthoff VA, Beuthien-Baumann B, Kalbe E, et al. Regional cerebral metabolism in early Alzheimer's disease with clinically significant apathy or depression. Biol Psychiatry. 2005;57(4):412-421.

13. Lee DY, Choo IH, Jhoo JH, et al. Frontal dysfunction underlies depressive syndrome in Alzheimer disease: a FDG-PET study. Am J Geriatr Psychiatry. 2006;14(7):625-628.

14. Hirono N, Mori E, Ishii K, et al. Frontal lobe hypometabolism and depression in Alzheimer's disease. Neurology. 1998;50(2):380-383.

15. Brendel M, Pogarell O, Xiong G, Delker A, Bartenstein P, Rominger A; Alzheimer's Disease Neuroimaging Initiative. Depressive symptoms accelerate cognitive decline in amyloid-positive MCI patients. Eur $J$ Nucl Med Mol Imaging. 2015;42(5):716-724.

16. van den Heuvel MP, Mandl RC, Kahn RS, Hulshoff Pol HE. Functionally linked resting-state networks reflect the underlying structural connectivity architecture of the human brain. Hum Brain Mapp. 2009; 30(10):3127-3141.

17. He Y, Chen Z, Gong G, Evans A. Neuronal networks in Alzheimer's disease. Neuroscientist. 2009;15(4):333-350.

18. Supekar K, Menon V, Rubin D, Musen M, Greicius MD. Network analysis of intrinsic functional brain connectivity in Alzheimer's disease. PLoS Comput Biol. 2008;4(6):e1000100.

19. Guo Z, Liu X, Jia X, et al. Regional coherence changes in Alzheimer's disease patients with depressive symptoms: a resting-state functional MRI study. J Alzheimers Dis. 2015;48(3):603-611.

20. Guo Z, Liu X, Hou H, Wei F, Liu J, Chen X. Abnormal degree centrality in Alzheimer's disease patients with depression: a resting-state functional magnetic resonance imaging study. Exp Gerontol. 2016;79:61-66.

21. Greicius MD, Krasnow B, Reiss AL, Menon V. Functional connectivity in the resting brain: a network analysis of the default mode hypothesis. Proc Natl Acad Sci U S A. 2003;100(1):253-258.

22. Zhang HY, Wang SJ, Liu B, et al. Resting brain connectivity: changes during the progress of Alzheimer disease. Radiology. 2010;256(2): 598-606.

23. Broyd SJ, Demanuele C, Debener S, Helps SK, James CJ, SonugaBarke EJ. Default-mode brain dysfunction in mental disorders: a systematic review. Neurosci Biobehav Rev. 2009;33(3):279-296.

24. Grimm S, Boesiger P, Beck J, et al. Altered negative BOLD responses in the default-mode network during emotion processing in depressed subjects. Neuropsychopharmacology. 2009;34(4):932-943.

25. Wu D, Yuan Y, Bai F, You J, Li L, Zhang Z. Abnormal functional connectivity of the default mode network in remitted late-onset depression. $J$ Affect Disord. 2013;147(1-3):277-287.

26. Zhong Y, Huang L, Cai S, et al. Altered effective connectivity patterns of the default mode network in Alzheimer's disease: an fMRI study. Neurosci Lett. 2014;578:171-175.

27. McKhann GM, Knopman DS, Chertkow H, et al. The diagnosis of dementia due to Alzheimer's disease: recommendations from the National Institute on Aging-Alzheimer's Association workgroups on diagnostic guidelines for Alzheimer's disease. Alzheimers Dement. 2011;7(3): 263-269.
28. Gmitrowicz A, Kucharska A. [Developmental disorders in the fourth edition of the American classification: diagnostic and statistical manual of mental disorders (DSM IV - optional book)]. Psychiatr Pol. 1994; 28(5):509-521. Polish.

29. Hamilton M. Development of a rating scale for primary depressive illness. Br J Soc Clin Psychol. 1967;6(4):278-296.

30. Cummings JL, Mega M, Gray K, Rosenberg-Thompson S, Carusi DA, Gornbein J. The Neuropsychiatric Inventory: comprehensive assessment of psychopathology in dementia. Neurology. 1994;44(12): $2308-2314$

31. Hirono N, Mori E, Ikejiri Y, et al. [Japanese version of the Neuropsychiatric Inventory - a scoring system for neuropsychiatric disturbance in dementia patients]. No to Shinkei. 1997;49(3):266-271. Japanese.

32. Schneider LS, Tariot PN, Lyketsos CG, et al. National Institute of Mental Health Clinical Antipsychotic Trials of Intervention Effectiveness (CATIE): Alzheimer disease trial methodology. Am J Geriatr Psychiatry. 2001;9(4):346-360.

33. Liu H, Liu Z, Liang M, et al. Decreased regional homogeneity in schizophrenia: a resting state functional magnetic resonance imaging study. Neuroreport. 2006;17(1):19-22.

34. Biswal B, Yetkin FZ, Haughton VM, Hyde JS. Functional connectivity in the motor cortex of resting human brain using echo-planar MRI. Magn Reson Med. 1995;34(4):537-541.

35. Maldjian JA, Laurienti PJ, Kraft RA, Burdette JH. An automated method for neuroanatomic and cytoarchitectonic atlas-based interrogation of fMRI data sets. NeuroImage. 2003;19(3):1233-1239.

36. Maldjian JA, Laurienti PJ, Burdette JH. Precentral gyrus discrepancy in electronic versions of the Talairach atlas. Neuroimage. 2004;21(1): 450-455.

37. Wang L, Zang Y, He Y, et al. Changes in hippocampal connectivity in the early stages of Alzheimer's disease: evidence from resting state fMRI. Neuroimage. 2006;31(2):496-504.

38. Wang S, Zhang Z, Lu G, Luo L. Localization of brain activity by temporal anti-correlation with the posterior cingulate cortex. Conf Proc IEEE Eng Med Biol Soc. 2007;2007:5227-5230.

39. Wu QZ, Li DM, Kuang WH, et al. Abnormal regional spontaneous neural activity in treatment-refractory depression revealed by restingstate fMRI. Hum Brain Mapp. 2011;32(8):1290-1299.

40. Desgranges B, Baron JC, Lalevee C, et al. The neural substrates of episodic memory impairment in Alzheimer's disease as revealed by FDG-PET: relationship to degree of deterioration. Brain. 2002;125 (Pt 5):1116-1124.

41. Eustache F, Desgranges B, Giffard B, de la Sayette V, Baron JC. Entorhinal cortex disruption causes memory deficit in early Alzheimer's disease as shown by PET. Neuroreport. 2001;12(4):683-685.

42. Aupee AM, Desgranges B, Eustache F, et al. Voxel-based mapping of brain hypometabolism in permanent amnesia with PET. Neuroimage. 2001;13(6 Pt 1):1164-1173.

43. Della-Maggiore V, Sekuler AB, Grady CL, Bennett PJ, Sekuler R, McIntosh AR. Corticolimbic interactions associated with performance on a short-term memory task are modified by age. J Neurosci. 2000; 20(22):8410-8416.

44. Allen G, Barnard H, McColl R, et al. Reduced hippocampal functional connectivity in Alzheimer disease. Arch Neurol. 2007;64(10): 1482-1487.

45. Eichenbaum H, Yonelinas AP, Ranganath C. The medial temporal lobe and recognition memory. Annu Rev Neurosci. 2007;30:123-152.

46. Phillips ML, Drevets WC, Rauch SL, Lane R. Neurobiology of emotion perception II: implications for major psychiatric disorders. Biol Psychiatry. 2003;54(5):515-528.

47. Seminowicz DA, Mayberg HS, McIntosh AR, et al. Limbic-frontal circuitry in major depression: a path modeling metanalysis. Neuroimage. 2004;22(1):409-418.

48. Kohen R, Kirov S, Navaja GP, et al. Gene expression profiling in the hippocampus of learned helpless and nonhelpless rats. Pharmacogenomics J. 2005;5(5):278-291. 
49. Lachman HM, Papolos DF, Weiner ED, et al. Hippocampal neuropeptide $\mathrm{Y}$ mRNA is reduced in a strain of learned helpless resistant rats. Brain Res Mol Brain Res. 1992;14(1-2):94-100.

50. LaBar KS, Cabeza R. Cognitive neuroscience of emotional memory. Nat Rev Neurosci. 2006;7(1):54-64.

51. Savitz J, Drevets WC. Bipolar and major depressive disorder: neuroimaging the developmental-degenerative divide. Neurosci Biobehav Rev. 2009;33(5):699-771.

52. Rapp MA, Schnaider-Beeri M, Grossman HT, et al. Increased hippocampal plaques and tangles in patients with Alzheimer disease with a lifetime history of major depression. Arch Gen Psychiatry. 2006; 63(2):161-167.

53. Aminoff EM, Kveraga K, Bar M. The role of the parahippocampal cortex in cognition. Trends Cogn Sci. 2013;17(8):379-390.

54. Eichenbaum H, Sauvage M, Fortin N, Komorowski R, Lipton P. Towards a functional organization of episodic memory in the medial temporal lobe. Neurosci Biobehav Rev. 2012;36(7):1597-1608.

55. Wang C, Stebbins GT, Medina DA, et al. Atrophy and dysfunction of parahippocampal white matter in mild Alzheimer's disease. Neurobiol Aging. 2012;33(1):43-52.

56. Dickerson BC, Feczko E, Augustinack JC, et al. Differential effects of aging and Alzheimer's disease on medial temporal lobe cortical thickness and surface area. Neurobiol Aging. 2009;30(3):432-440.

57. Brugnolo A, Morbelli S, Dessi B, et al. The reversed clock drawing test phenomenon in Alzheimer's disease: a perfusion SPECT study. Dement Geriatr Cogn Disord. 2010;29(1):1-10.

58. Chen G, Ward BD, Chen G, Li SJ. Decreased effective connectivity from cortices to the right parahippocampal gyrus in Alzheimer's disease subjects. Brain Connect. 2014;4(9):702-708.

59. Liu J, Zhang X, Yu C, et al. Impaired parahippocampus connectivity in mild cognitive impairment and Alzheimer's disease. J Alzheimers Dis. 2015;49(4):1051-1064

60. Arnone D, Job D, Selvaraj S, et al. Computational meta-analysis of statistical parametric maps in major depression. Hum Brain Mapp. 2016;37(4):1393-1404.

61. Canu E, Kostic M, Agosta F, et al. Brain structural abnormalities in patients with major depression with or without generalized anxiety disorder comorbidity. J Neurol. 2015;262(5):1255-1265.

62. Zamoscik V, Huffziger S, Ebner-Priemer U, Kuehner C, Kirsch P. Increased involvement of the parahippocampal gyri in a sad mood predicts future depressive symptoms. Soc Cogn Affect Neurosci. 2014;9(12): 2034-2040.

63. Zeng LL, Shen H, Liu L, et al. Identifying major depression using whole-brain functional connectivity: a multivariate pattern analysis. Brain. 2012;135(Pt 5):1498-1507.

64. Ongur D, Ferry AT, Price JL. Architectonic subdivision of the human orbital and medial prefrontal cortex. J Comp Neurol. 2003;460(3): 425-449.

65. Sheline YI, Price JL, Yan Z, Mintun MA. Resting-state functional MRI in depression unmasks increased connectivity between networks via the dorsal nexus. Proc Natl Acad Sci U S A. 2010;107(24): 11020-11025.

66. Kim SM, Park SY, Kim Y, et al. Affective network and default mode network in depressive adolescents with disruptive behaviors. Neuropsychiatr Dis Treat. 2016;12:49-56.

67. Dolan RJ, Lane R, Chua P, Fletcher P. Dissociable temporal lobe activations during emotional episodic memory retrieval. Neuroimage. 2000;11(3):203-209.

68. Price JL, Drevets WC. Neurocircuitry of mood disorders. Neuropsychopharmacology. 2010;35(1):192-216.

69. Suslow T, Konrad C, Kugel H, et al. Automatic mood-congruent amygdala responses to masked facial expressions in major depression. Biol Psychiatry. 2010;67(2):155-160.

70. Wang L, LaBar KS, Smoski M, et al. Prefrontal mechanisms for executive control over emotional distraction are altered in major depression. Psychiatry Res. 2008;163(2):143-155.
71. Ramasubbu R, Konduru N, Cortese F, Bray S, Gaxiola-Valdez I, Goodyear B. Reduced intrinsic connectivity of amygdala in adults with major depressive disorder. Front Psychiatry. 2014;5:17.

72. Avery JA, Drevets WC, Moseman SE, Bodurka J, Barcalow JC, Simmons WK. Major depressive disorder is associated with abnormal interoceptive activity and functional connectivity in the insula. Biol Psychiatry. 2014;76(3):258-266.

73. Ho TC, Yang G, Wu J, et al. Functional connectivity of negative emotional processing in adolescent depression. J Affect Disord. 2014; 155:65-74.

74. Yao H, Zhou B, Zhang Z, et al. Longitudinal alteration of amygdalar functional connectivity in mild cognitive impairment subjects revealed by resting-state FMRI. Brain Connect. 2014;4(5):361-370.

75. Hamann SB, Monarch ES, Goldstein FC. Memory enhancement for emotional stimuli is impaired in early Alzheimer's disease. Neuropsychology. 2000;14(1):82-92.

76. Yao H, Liu Y, Zhou B, et al. Decreased functional connectivity of the amygdala in Alzheimer's disease revealed by resting-state fMRI. Eur J Radiol. 2013;82(9):1531-1538.

77. Olson IR, Plotzker A, Ezzyat Y. The enigmatic temporal pole: a review of findings on social and emotional processing. Brain. 2007; 130(Pt 7):1718-1731.

78. Mathiak KA, Klasen M, Weber R, Ackermann H, Shergill SS, Mathiak K. Reward system and temporal pole contributions to affective evaluation during a first person shooter video game. BMC Neurosci. 2011;12:66.

79. Shin LM, Dougherty DD, Orr SP, et al. Activation of anterior paralimbic structures during guilt-related script-driven imagery. Biol Psychiatry. 2000;48(1):43-50.

80. Kimbrell TA, George MS, Parekh PI, et al. Regional brain activity during transient self-induced anxiety and anger in healthy adults. Biol Psychiatry. 1999;46(4):454-465.

81. Tillfors M, Furmark T, Marteinsdottir I, et al. Cerebral blood flow in subjects with social phobia during stressful speaking tasks: a PET study. Am J Psychiatry. 2001;158(8):1220-1226.

82. Beauregard M, Paquette V, Levesque J. Dysfunction in the neural circuitry of emotional self-regulation in major depressive disorder. Neuroreport. 2006;17(8):843-846.

83. Guo W, Liu F, Liu J, et al. Increased cerebellar-default-mode-network connectivity in drug-naive major depressive disorder at rest. Medicine (Baltimore). 2015;94(9):e560.

84. Liu L, Zeng LL, Li Y, et al. Altered cerebellar functional connectivity with intrinsic connectivity networks in adults with major depressive disorder. PloS One. 2012;7(6):e39516.

85. Xie C, Bai F, Yu H, et al. Abnormal insula functional network is associated with episodic memory decline in amnestic mild cognitive impairment. Neuroimage. 2012;63(1):320-327.

86. Lehmann M, Crutch SJ, Ridgway GR, et al. Cortical thickness and voxel-based morphometry in posterior cortical atrophy and typical Alzheimer's disease. Neurobiol Aging. 2011;32(8):1466-1476.

87. Yakushev I, Schreckenberger M, Muller MJ, et al. Functional implications of hippocampal degeneration in early Alzheimer's disease: a combined DTI and PET study. Eur J Nucl Med Mol Imaging. 2011; 38(12):2219-2227.

88. Leech R, Sharp DJ. The role of the posterior cingulate cortex in cognition and disease. Brain. 2014;137(Pt 1):12-32.

89. Eklund A, Nichols TE, Knutsson H. Cluster failure: why fMRI inferences for spatial extent have inflated false-positive rates. Proc Natl Acad Sci U S A. 2016;113(28):7900-7905.

90. Strother SC. Evaluating fMRI preprocessing pipelines. IEEE Eng Med Biol Mag. 2006;25(2):27-41.

91. Murphy K, Birn RM, Handwerker DA, Jones TB, Bandettini PA. The impact of global signal regression on resting state correlations: are anticorrelated networks introduced? Neuroimage. 2009;44(3):893-905.

92. Cummings JL. The Neuropsychiatric Inventory: assessing psychopathology in dementia patients. Neurology. 1997;48(5 Suppl 6):S10-S16. 
93. Binetti G, Mega MS, Magni E, et al. Behavioral disorders in Alzheimer disease: a transcultural perspective. Arch Neurol. 1998;55(4): 539-544.

94. Cummings JL. Theories behind existing scales for rating behavior in dementia. Int Psychogeriatr. 1996;8 Suppl 3:293-300.
95. Becker JT, Boller F, Lopez OL, Saxton J, McGonigle KL. The natural history of Alzheimer's disease. Description of study cohort and accuracy of diagnosis. Arch Neurol. 1994;51(6):585-594.

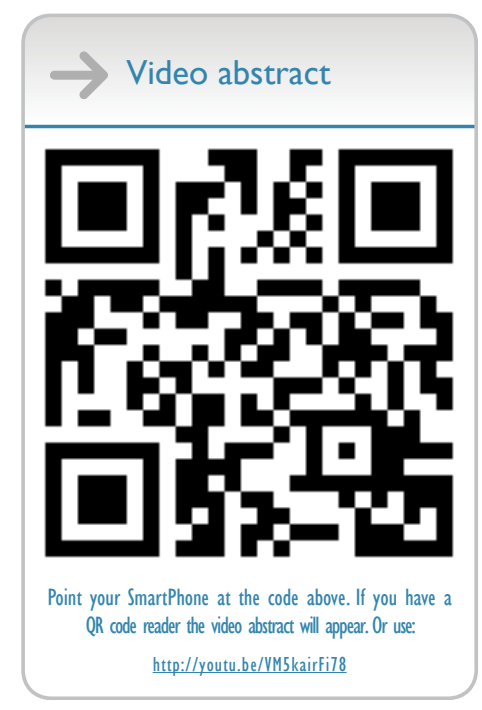

Neuropsychiatric Disease and Treatment

Dovepress

\section{Publish your work in this journal}

Neuropsychiatric Disease and Treatment is an international, peerreviewed journal of clinical therapeutics and pharmacology focusing on concise rapid reporting of clinical or pre-clinical studies on a range of neuropsychiatric and neurological disorders. This journal is indexed on PubMed Central, the 'PsycINFO' database and CAS,

and is the official journal of The International Neuropsychiatric Association (INA). The manuscript management system is completely online and includes a very quick and fair peer-review system, which is all easy to use. Visit http://www.dovepress.com/testimonials.php to read real quotes from published authors. 